\title{
DIFERENCIA DE PRESENTACIÓN DEL SÍNDROME CORONARIO AGUDO POR GÉNERO EN PACIENTES LLEVADOS AL LABORATORIO DE CATETERISMO EN POBLACIÓN DOMINICANA: UN ESTUDIO RETROSPECTIVO
}

\section{Acute Coronary Syndrome presentation difference by gender in patients taken to catheterization laboratory in Dominican population: A retrospective study}

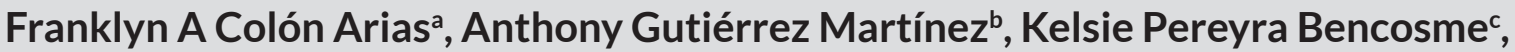 \\ Aimée Mercado Domínguez ${ }^{\mathrm{d}}$, Francisco Gutiérrez Lora ${ }^{\mathrm{e}}$, Maribel Almonte Casado ${ }^{f}$ \\ y José Rafael López Luciano"
}

Recibido: 2 de mayo, $2020 \bullet$ Aprobado: 6 de agosto, 2020

Cómo citar: Colón Arias FA, Martínez AG, Pereyra Bencosme K, Mercado Domínguez A, Gutiérrez Lora F, Almonte Casado M, López Luciano JR. Diferencia de presentación del síndrome coronario agudo por género en pacientes llevados al laboratorio de cateterismo en población dominicana: un estudio retrospectivo. cysa [Internet]. 9 de junio de 2021 [citado 16 de junio de 2021];5(2):69-6. Disponible en: https://revistas.intec.edu.do/index.php/cisa/article/view/2213

\section{Resumen}

Introducción: el síndrome coronario agudo (SCA) es una serie de signos y síntomas que se refieren a isquemia miocárdica repentina. Estas condiciones clínicas son Síndrome Coronario agudo sin Elevación del Segmento ST (SCASEST) y Síndrome Coronario Agudo con Elevación del Segmento ST (SCACEST). A nivel mundial hay hallazgos controversiales con respecto a la diferencia de presentación de SCA en hombres y mujeres.

Objetivo: determinar las diferencias en presentación del síndrome coronario agudo según el género en República Dominicana.

Métodos: en este estudio observacional retrospectivo unicéntrico, 3,548 pacientes con SCA llevados al laboratorio de cateterismo fueron observados durante el período

\footnotetext{
a Doctor en Medicina. Corazones del Cibao, Santiago de los Caballeros, República Dominicana. ORCID: 0000-0001-53

Correo-e: fcolon6322@gmail.com

b Doctor en Medicina. Pontificia Universidad Católica Madre y Maestra, Facultad Ciencias de la Salud, Escuela de Medicina, Santiago de los Caballeros, República Dominicana. ORCID: 0000-0003-4567-0123, Correo-e: AnthJGutierrez@gmail.com
}

\begin{abstract}
Introduction: Acute Coronary Syndrome (ACS) is a series of signs and symptoms referring to sudden myocardial ischemia. These clinical conditions are: Non-ST-Acute Coronary Syndromes (NST-ACS) and ST-segment Elevation Myocardial Infarction (STEMI). Globally there are controversial findings regarding the difference in SCA presentation in both men and women.

Objective: Determine differences in presentation of acute coronary syndrome by gender in the Dominican Republic.

Methods: In this retrospective single-centered observational study 3,548 patients with ACS taken to the catheterization laboratory underwent observation, during the time

\footnotetext{
${ }^{\mathrm{c}}$ Doctora en Medicina. Corazones del Cibao, Santiago de los Caballeros, República Dominicana. ORCID: 0000-0002-5195-4483 Correo-e: kelsiepereyra@gmail.com

d Doctora en Medicina. Clínica Corominas, Santiago de los Caballeros, República Dominicana. Correo-e: aimeemercadod@gmail.com

e Doctor en Medicina. Corazones del Cibao, Santiago de los Caballeros, República Dominicana. Correo-e: fagl_17@hotmail.com

${ }_{\mathrm{f}}$ Doctora en Medicina. Corazones del Cibao, Santiago de los Caballeros, República Dominicana. Correo-e: dra.almontecasado@gmail.com

g Doctor en Medicina. Corazones del Cibao, Santiago de los Caballeros, República Dominicana. Correo-e: corazonesdelcibao@yahoo.com
} 
de agosto de 2016 a septiembre de 2019.

Resultados: la edad promedio de presentación fue mayor en las mujeres, con una media de 63 años (DE \pm 12.2$)$. El SCACEST fue más frecuente en hombres $(69.35 \%)$ y la Angina Inestable en mujeres $(50.14 \%, \mathrm{p}<0.0001)$. Angiográficamente, la enfermedad coronaria multivascular fue más común en hombres y la angiografía coronaria normal en mujeres $(41.30 \%$ y $35.37 \%$, p $<0.0001)$.

Conclusión: dentro de la población dominicana, la mujer tiende a ser mayor al momento de presentación de SCA, con menor tendencia a someterse a intervención coronaria terapéutica.

Palabras clave: cardiología intervencionista; síndrome coronario agudo; riesgo cardiovascular; evento cardiovascular; República Dominicana.

\section{Introducción}

SCA es la manifestación clínica característica de la arteriopatía coronaria $(\mathrm{AC})$ y los factores de riesgo más prevalentes asociados con AC y SCA son tabaquismo, hipercolesterolemia, hiperglucemia, hipertensión, IMC> $25 \mathrm{~kg} / \mathrm{m}^{2}$ y antecedentes familiares de $A^{1}$. La mayoría de estos son modificables o tratables para mantener un control adecuado. La asociación entre estos factores se ha tenido en cuenta al estudiar el SCA. Acerca de esto, la literatura muestra que en el momento de la presentación del SCA, las mujeres tienen una menor prevalencia de tabaquismo, pero mayores riesgos cardiovasculares de diabetes e hipercolesterolemia ${ }^{2,3}$.

En cuanto al género, su asociación con la edad de presentación de SCA es controversial. Varios estudios muestran que las mujeres componen un tercio de la población que presenta $\mathrm{SCA}^{4,5,6}$, lo que también demuestra una tendencia creciente con la edad, tanto para hombres como para mujeres. En el momento de la presentación, las mujeres tienden a ser mayores que los hombres y tienen múltiples comorbilidades ${ }^{3,4}$. Esto apoya una mayor mortalidad intrahospitalaria en las mujeres en comparación con los hombres, frame of August 2016 to September 2019.

Results: The average age of presentation was higher in females, with a mean of 63 years old $(S D \pm 12.2)$. STEMI was greater in males $(69.35 \%)$ and females presented more with UA (50.14\%, p<0.0001). Angiography findings showed that multi-vascular coronary artery disease was more common in males and normal coronary angiography more frequent in females $(41.30 \%$ and $35.37 \%$, $\mathrm{p}<0.0001)$.

Conclusion: Within the Dominican population, females tend to be older at the time of appearance of ACS with a lower tendency of undergoing coronary intervention.

Keywords: Interventional cardiology; acute coronary syndrome; cardiovascular risk; cardiovascular event; Dominican Republic.

siendo las comorbilidades la posible causa ${ }^{4}$. Angiográficamente, las mujeres son propensas a presentar lesiones de un solo vaso, teniendo menos presentaciones de SCACEST como una condición de debut en contraste con los hombres ${ }^{7,8}$.

Con tantas disparidades en los datos globales y la ausencia absoluta de descripciones locales, este estudio tiene como objetivo determinar las diferencias de género en la prevalencia, epidemiología, presentación clínica, hallazgos angiográficos y resultado de los pacientes con SCA ingresados para angiografía diagnóstica y/o terapéutica en Santiago de los Caballeros, República Dominicana, desde agosto de 2016 hasta septiembre de 2019.

\section{Métodos}

Esta investigación llevó a cabo una observación retrospectiva monocéntrica de 3,548 casos desde agosto del 2016 hasta septiembre del 2019 en Santiago de los Caballeros, República Dominicana. El registro de datos comenzó desde la admisión hasta la salida del centro de salud, por parte de un médico de servicio, que aseguró los datos en un servidor privado exclusivo del departamento de cardiología. 
La base de datos tiene cada paciente evaluado por la unidad de cardiología intervencionista; de este grupo, incluimos aquellos casos con un diagnóstico definitivo de síndrome coronario agudo y llevados al quirófano de cardiología intervencionista. La información sociodemográfica, clínica, angiográfica y de procedimiento se requirió para este estudio.

La obtención y el análisis de la información fue realizada por diferentes miembros del equipo de investigación para evitar sesgos de selección. Las variables continuas fueron descritas como media y desviación estándar, las variables categóricas se basan en el recuento y el porcentaje con la prueba de T-student y Chi-cuadrado para el contraste de hipótesis, respectivamente, considerando estadísticamente significativo un valor de $\mathrm{P}<0.05$. En cuanto a los datos faltantes, consideramos solo casos completos. STATA15 se utilizó como software de análisis estadístico.

\section{Resultados}

La muestra consistió en $57.05 \%$ hombres y $42.95 \%$ mujeres con una edad promedio al momento de la presentación de 63.04 (DE \pm 12.35$)$ años. Además, las mujeres tenían una edad promedio más alta en contraste con los hombres con 63.89 (DE \pm 12.20 ) ańos y $62.41(\mathrm{DE} \pm 12.42)$ ańos, respectivamente. En cuanto a los antecedentes médicos, el 78.72 $\%$ de la muestra tenía hipertensión arterial sistémica, el $16.10 \%$ tenía dislipidemia, el $0.48 \%$ eran diabéticos tipo 1, mientras que el $34.53 \%$ eran diabéticos tipo 2 y el $3.10 \%$ sufría insuficiencia cardíaca. Igualmente, la evaluación del consumo de tabaco reveló que el 31.09 \% eran fumadores anteriores, mientras que el $10.85 \%$ eran consumidores actuales. La presentación más frecuente de SCA en la población dada fue SCASEST con $75.17 \%$ del diagnóstico. De los procedimientos realizados, el 93.12 \% utilizó la arteria Radial Derecha como acceso y el 52.65 \% progresó a angioplastía percutánea trasluminal coronaria. Además, la enfermedad de múltiples vasos fue el resultado de coronarografía más frecuente, con el $40.95 \%$ de los casos, y el vaso más afectado, con una frecuencia de compromiso del $53.78 \%$, fue la arteria descendente anterior. La mortalidad intrahospitalaria se reportó en el $1.66 \%$ de los casos. (Véase la tabla 1 para obtener más detalles sobre la descripción de la muestra).

Hubo superioridad numérica de la hipertensión arterial sistémica, dislipidemia, insuficiencia cardíaca $\mathrm{y}$ antecedentes de tabaquismo en los hombres, en contraste con DM tipo 1 y 2 que fueron más recurrentes en las mujeres. La asociación de DM tipo 1 con las mujeres fue estadísticamente significativa $(\mathrm{p}=$ 0.021). Además, hubo una diferencia en la presentación clínica de SCA, siendo SCACEST y SCASEST numéricamente superiores en hombres (69.35\% y $64.10 \%$, de manera respectiva) en comparación con las mujeres, que presentaron una asociación estadísticamente significativa con AI $(50.14 \%$, p $<0.0001)$. En cuanto a los resultados de la angiografía, los hombres tenían enfermedad arterial coronaria multivascular más compleja y en las mujeres las arterias coronarias normales eran más habituales (41.30 \% y $35.37 \%$, respectivamente, p <0.0001). Cuando se contabilizaron vasos específicos, la arteria descendiente anterior y la arteria circunfleja se vieron más afectadas en los hombres ( $p<0.001$ y $\mathrm{p}<0.011$ ), junto con más intervenciones terapéuticas en general en este género (59.58 \%, p <0.0001). La tasa de mortalidad dentro del laboratorio de cateterismo reveló una relación numérica no estadísticamente significativa que favorece a las mujeres sobre los hombres. (Véase tabla 2). El número de vasos comprometidos evaluados junto con la presencia de comorbilidades arrojó que la insuficiencia cardíaca y la dislipidemia muestran una asociación estadísticamente significativa con la enfermedad de múltiples vasos ( $\mathrm{p} 0.031$ y p <0.001, respectivamente), mientras que la hipertensión arterial sistémica, DM tipo 1 y 2 y el consumo de tabaco, ya sea consumo previo o actual, fue más frecuente en pacientes con enfermedad de múltiples vasos, aunque no estadísticamente significativo. (Véase tabla 3). 

Franklyn A Colón Arias, Anthony Gutiérrez Martínez, Kelsie Pereyra Bencosme, Aimée Mercado Domínguez,
Francisco Gutiérrez Lora, Maribel Almonte Casado y José Rafael López Luciano

Tabla 1. Descripción clínica y sociodemográfica de la muestra

\begin{tabular}{|c|c|c|}
\hline \multicolumn{2}{|l|}{ Variable } & Media (DE) \\
\hline \multicolumn{2}{|l|}{ Edad (ańos) } & $63.04( \pm 12.35)$ \\
\hline \multicolumn{2}{|l|}{ Hombre } & $62.41( \pm 12.42)$ \\
\hline \multicolumn{2}{|l|}{ Mujer } & $63.89( \pm 12.20)$ \\
\hline Variable & Categoría & N (\%) \\
\hline \multirow{2}{*}{ Género } & Hombre & $2,024(57.05 \%)$ \\
\hline & Mujer & $1,524(42.95 \%)$ \\
\hline \multirow{3}{*}{ Diagnóstico } & SCACEST & $881(24.83 \%)$ \\
\hline & SCASEST & $585(16.49 \%)$ \\
\hline & Angina inestable & $2,082(58.68 \%)$ \\
\hline \multirow{2}{*}{ Tratamiento } & Angiografía diagnóstica & $1,680(47.35 \%)$ \\
\hline & Angioplastía coronaria & $1,868(52.65 \%)$ \\
\hline \multirow{3}{*}{ Acceso angiográfico } & Radial derecha & $3,304(93.12 \%)$ \\
\hline & Radial izquierda & $117(3.30 \%)$ \\
\hline & Femoral derecha & $127(3.58 \%)$ \\
\hline \multirow{4}{*}{ Número de vasos comprometidos } & Arterias normales & $1,017(28.66 \%)$ \\
\hline & Una arteria (1) & $569(16.04 \%)$ \\
\hline & Dos arterias (2) & $509(14.35 \%)$ \\
\hline & Multivascular $(\geq 3)$ & $1,453(40.95 \%)$ \\
\hline \multirow{2}{*}{ Mortalidad intrahospitalaria } & Sí & $59(1.66 \%)$ \\
\hline & No & $3,489(98.34 \%)$ \\
\hline \multirow{2}{*}{ Hipertensión } & Sí & $2,793(78.72 \%)$ \\
\hline & No & $755(21.28 \%)$ \\
\hline \multirow{2}{*}{ Dislipidemia } & Sí & $571(16.10 \%)$ \\
\hline & No & $2,976(83.90 \%)$ \\
\hline \multirow{2}{*}{ Diabetes tipo 1} & Sí & $17(0.48 \%)$ \\
\hline & No & $3,531(99.52 \%)$ \\
\hline \multirow{2}{*}{ Diabetes tipo 2} & Sí & $1,225(34.53 \%)$ \\
\hline & No & $2,323(65.47 \%)$ \\
\hline \multirow{2}{*}{ Consumo de tabaco previo } & Sí & $1,103(31.09 \%)$ \\
\hline & No & $2,445(68.91 \%)$ \\
\hline \multirow{2}{*}{ Consumo de tabaco actual } & Sí & $385(10.85 \%)$ \\
\hline & No & $3,163(89.15 \%)$ \\
\hline \multirow{2}{*}{ Fallo cardíaco } & Sí & $110(3.10 \%)$ \\
\hline & No & $3,438(96.90 \%)$ \\
\hline \multicolumn{3}{|c|}{ Compromiso vascular por arteria } \\
\hline \multicolumn{2}{|c|}{ Descendente anterior } & $1,908(53.78 \%)$ \\
\hline \multicolumn{2}{|c|}{ Coronaria derecho } & $1,543(43.49 \%)$ \\
\hline \multicolumn{2}{|c|}{ Arteria circunfleja } & $1,280(36.08 \%)$ \\
\hline \multicolumn{2}{|c|}{ Descendente posterior } & $168(4.74 \%)$ \\
\hline \multicolumn{2}{|c|}{ Obtusa marginal } & $415(11.70 \%)$ \\
\hline \multicolumn{2}{|c|}{ Tronco coronario izquierdo } & $387(10.91 \%)$ \\
\hline
\end{tabular}

72 | Ciencia y Salud 2021; 5(2, mayo-agosto): 69-76• Artículo original 
SCACEST = Síndrome Coronario Agudo sin Elevación del Segmento ST, SCASEST = Síndrome Coronario Agudo con Elevación del Segmento ST

La tabla 1 muestra los valores sociodemográficos de la población. La edad promedio fue mayor en las mujeres y los hombres fueron numéricamente predominantes. Además, el diagnóstico más común fue angina inestable, seguida de SCACEST. Alrededor del $53 \%$ de los pacientes requirieron angioplastía coronaria, siendo más frecuente el abordaje a través de la arteria radial derecha. El hallazgo principal fue la enfermedad de múltiples vasos, definida como tres o más vasos comprometidos.

Tabla 2. Análisis estadístico por género

\begin{tabular}{|c|c|c|c|c|}
\hline Variable & Hombre (conteo y \%) & Mujer (conteo y \%) & Total (n) & Valor $\mathrm{p}$ \\
\hline Hipertensión & $1,608(57.57 \%)$ & $1,185(42.43 \%)$ & 2,793 & 0.223 \\
\hline Diabetes tipo 2 & $693(56.57 \%)$ & $532(43.43 \%)$ & 1,225 & 0.678 \\
\hline Diabetes tipo 1 & $5(29.41 \%)$ & $12(70.59 \%)$ & 17 & 0.021 \\
\hline Dislipidemia & $330(57.79 \%)$ & $241(42.21 \%)$ & 571 & 0.689 \\
\hline Consumo previo de tabaco & $614(55.67 \%)$ & $489(44.33 \%)$ & 1,103 & 0.265 \\
\hline Consumo actual de tabaco & $218(56.62 \%)$ & $167(43.38 \%)$ & 385 & 0.859 \\
\hline Fallo cardíaco & $64(58.18 \%)$ & $46(41.82 \%)$ & 110 & 0.807 \\
\hline Mortalidad & $31(52.54 \%)$ & $28(47.46 \%)$ & 59 & 0.481 \\
\hline \multicolumn{5}{|c|}{ Acceso angiográfico } \\
\hline Radial derecha & $1,899(57.48 \%)$ & $1,405(42.52 \%)$ & 3,304 & \multirow{3}{*}{0.097} \\
\hline Radial izquierda & $56(47.86 \%)$ & $61(52.14 \%)$ & 117 & \\
\hline Femoral derecha & $69(54.33 \%)$ & $58(45.67 \%)$ & 127 & \\
\hline \multicolumn{5}{|c|}{ Diagnóstico } \\
\hline SCACEST & $611(69.35 \%)$ & $270(30.65 \%)$ & 881 & \multirow{3}{*}{$<0.001$} \\
\hline SCASEST & $375(64.10 \%)$ & $210(35.90 \%)$ & 585 & \\
\hline Angina inestable & $1,038(49.86 \%)$ & $1,044(50.14 \%)$ & 2,082 & \\
\hline \multicolumn{5}{|c|}{ Tratamiento } \\
\hline Angiografía diagnóstica & $818(48.69 \%)$ & $862(51.31 \%)$ & 1,680 & \multirow{2}{*}{$<0.001$} \\
\hline Angioplastía coronaria & $1,206(64.56 \%)$ & $662(35.44 \%)$ & 1,868 & \\
\hline \multicolumn{5}{|c|}{ Número de vasos comprometidos } \\
\hline Coronarias normales & $478(47.00 \%)$ & $539(53.00 \%)$ & 1,017 & \multirow{4}{*}{$<0.001$} \\
\hline Una arteria & $365(64.15 \%)$ & $204(35.85 \%)$ & 569 & \\
\hline Dos arterias & $345(67.78 \%)$ & $164(32.22 \%)$ & 509 & \\
\hline Multivascular $(\geq 3)$ & $836(57.54 \%)$ & $617(57.54 \%)$ & 1,453 & \\
\hline \multicolumn{5}{|c|}{ Compromiso vascular por arteria } \\
\hline Descendente anterior & $1,137(59.59 \%)$ & $771(40.41 \%)$ & 1,908 & 0.001 \\
\hline Coronaria derecha & $907(58.78 \%)$ & $636(41.22 \%)$ & 1,543 & 0.067 \\
\hline Arteria circunfleja & $766(59.84 \%)$ & $514(40.16 \%)$ & 1,280 & 0.011 \\
\hline Descendiente posterior & $101(60.12 \%)$ & $67(39.88 \%)$ & 168 & 0.410 \\
\hline Obtusa marginal & $249(60.00 \%)$ & $166(40.00 \%)$ & 415 & 0.196 \\
\hline Tronco coronario izquierdo & $224(57.88 \%)$ & $163(42.12 \%)$ & 387 & 0.721 \\
\hline
\end{tabular}


La tabla 2 muestra cómo se distribuyen las variables por género. La mayoría de los antecedentes mórbidos no tuvieron una relación significativa con el compromiso vascular, salvo la presencia de diabetes tipo 1 en el sexo femenino. Lo mismo ocurre con el acceso arterial, donde el enfoque de hombres y mujeres fue similar. En cuanto a los hallazgos angiográficos y el tratamiento, la necesidad de angioplastia fue mayor en los hombres $(\mathrm{p}<0,001)$. También se observó esta tendencia en el predominio de SCACEST y SCASEST en hombres, mientras que las mujeres presentaron más AI.

Tabla 3. Análisis estadístico de comorbilidades por número de vasos comprometidos

\begin{tabular}{|c|c|c|c|c|c|}
\hline Variable & $\begin{array}{c}\text { Arterias normales } \\
\text { (conteo) }\end{array}$ & $\begin{array}{c}\text { Una arteria } \\
\text { (conteo) }\end{array}$ & $\begin{array}{c}\text { Dos arterias } \\
\text { (conteo) }\end{array}$ & $\begin{array}{c}\text { Multivascular } \\
\text { (conteo) }\end{array}$ & Valor p \\
\hline Hipertensión & 794 & 451 & 404 & 1,144 & 0.923 \\
\hline Diabetes tipo 1 & 5 & 4 & 1 & 7 & 0.693 \\
\hline Diabetes tipo 2 & 333 & 192 & 170 & 530 & 0.229 \\
\hline Consumo previo de tabaco & 312 & 179 & 139 & 473 & 0.174 \\
\hline Consumo actual de tabaco & 114 & 74 & 48 & 149 & 0.216 \\
\hline Fallo cardíaco & 24 & 16 & 26 & 44 & $\mathbf{0 . 0 3 1}$ \\
\hline Dislipidemia & 205 & 89 & 64 & 213 & $<\mathbf{0 . 0 0 1}$ \\
\hline
\end{tabular}

En cuanto a la tabla 3, una gran proporción de pacientes tiene enfermedad de múltiples vasos con presencia de comorbilidades, especialmente aquellos con dislipidemia e insuficiencia cardíaca, quienes obtuvieron significancia estadística previo a los demás antecedentes mórbidos.

\section{Discusión}

Extensos datos de investigación en todo el mundo exploran el SCA entre géneros, evidenciando resultados contradictorios. Actualmente, la tendencia de disminución en la mortalidad beneficia a los hombres sobre las mujeres ${ }^{9}$, mostrando un contraste biológico detrás de estos eventos. Un menor diámetro de las arterias coronarias en las mujeres puede contribuir a este fenómeno de interrupción repentina de la perfusión miocárdica y la presentación clínica atípica en comparación con los hombres, incluso después de ajustar por peso corporal o la masa ventricular en cada caso $^{10}$.

En la muestra estudiada, los hombres tienen superioridad numérica de hipertensión arterial sistémica,
DM tipo 2, dislipidemia e historial de tabaquismo, contrastando con estudios anteriores que atribuyen una mayor incidencia de hipertensión y DM tipo 2 a las mujeres ${ }^{11}$. Lo que lleva a reflexionar si el intenso escrutinio de riesgos y medidas de atención primaria son tan eficaces en las mujeres en comparación a los hombres a lo largo de estas últimas décadas ${ }^{12}$.

La AI como presentación clínica muestra significancia estadística favoreciendo a la población femenina, de acuerdo con datos anteriores que las describen como dos tercios de las presentaciones de $\mathrm{AI}^{13}$. Del mismo modo, pueden ser propensas a complicaciones debido a la tendencia a retrasar la búsqueda de atención médica hasta mucho después tras la aparición de los síntomas ${ }^{14}$. Angiográficamente, los hombres presentan más daño multivascular que las mujeres, como se reporta en la literatura ${ }^{15,16}$; en contraste, las mujeres mostraron más resultados de angiografía no patológica ${ }^{16}$. Las intervenciones coronarias entre géneros es un tema complejo, ya que la mayoría de las observaciones retrospectivas se basan en una proporción masculino:femenino desigual, con los hombres necesitando más inter- 
vención terapéutica, como se observa en esta investigación y en anteriores ${ }^{16}$.

En cuanto a las limitaciones de la investigación, la muestra se limita a los casos con diagnóstico de SCA que recibieron un enfoque invasivo, mientras que los pacientes con el mismo diagnóstico que fueron tratados conservadoramente no fueron tomados en cuenta. Además, la falta de datos locales de este tipo de sujetos limita la observación a una experiencia de un centro de salud. Los pacientes con SCA se benefician de seguimiento para determinar los resultados clínicos adecuados y la mortalidad a lo largo del tiempo, sin embargo, no hay medios de obtener estos datos dentro de un enfoque costo-efectivo. Además, la insuficiencia de comunicación entre los centros de salud hace imposible estimar el tiempo entre el inicio de los síntomas y la llegada al centro de salud/laboratorio de cateterismo.

\section{Conclusión}

República Dominicana parece tener hallazgos similares respecto al SCA y el género, mostrando una edad superior al momento de la presentación para las mujeres, así como patrones de riesgo distintos, características clínicas atípicas, mejores resultados angiográficos y menos necesidad de intervención terapéutica respecto a los hombres.

Como una población recientemente descrita, el equipo de investigación espera inducir a los investigadores locales a explotar esta información para explorar nuevos horizontes en cardiología y atención primaria en la comunidad latinoamericana debido a su homogeneidad con la muestra estudiada. Del mismo modo, recomendamos estudios prospectivos que utilicen estas conclusiones.

\section{Agradecimientos}

A la Clínica Corominas por el apoyo constante a la investigación clínica como herramienta para el avance constante de la medicina.
A la Pontificia Universidad Católica Madre y Maestra por la educación crítica que induce a sus alumnos a prosperar a través de las ciencias.

\section{- $\quad$ Financiamiento}

Esta investigación no recibió ninguna subvención específica de parte de alguna agencia de financiación en los sectores público, comercial o sin fines de lucro.

\section{Referencias}

1. Kumar A, Cannon CP.Acute coronary syndromes: diagnosis and management, part I. InMayo Clinic Proceedings. 2009;84(10):917-38. Elsevier.

2. Ten HaafME, Bax M, Ten Berg JM etal. Sex differences in characteristics and outcome in acute coronary syndrome patients in the Netherlands. Netherlands Heart Journal. 2019;27(5):263-71.

3. Duan JG, Chen XY, Wang L, Lau A et al. Sex differences in epidemiology and risk factors of acute coronary syndrome in Chinese patients with type 2 diabetes: a long-term prospective cohort study. PLoS One. 2015;10(4).

4. Worrall-Carter L, McEvedy S, Wilson A et al. Gender differences in presentation, coronary intervention, and outcomes of 28,985 acute coronary syndrome patients in Victoria, Australia. Women's Health Issues. 2016;26(1):14-20.

5. Pelletier R, Khan NA, Cox Jb et al. Sex versus gender-related characteristics: which predicts outcome after acute coronary syndrome in the young? Journal of the American College of Cardiology. 2016;67(2):127-35.

6. Stähli BE, Wischnewsky MB, Jakob $\mathrm{P}$ et al. Gender and age differences in outcomes of patients with acute coronary syndromes referred for coronary angiography. Catheterization and cardiovascular interventions. 2019;93(1):16-24. 
7. Khamis RY, Ammari T, Mikhail GW. Gender differences in coronary heart disease. Heart. 2016;102(14):1142-9.

8. Lorenzo AD. Gender Disparities and Outcomes of Acute Coronary Syndromes in Brazil. Arquivos brasileiros de cardiologia. 2018;111(5):654-5.

9. Gupta A, Wang Y, Spertus JA et al. Trends in acute myocardial infarction in young patients and differences by sex and race, 2001 to 2010 . J Am Coll Cardiol 2014;64:337-45.

10. Hiteshi AK, Li D, Gao Y et al. Gender differences in coronary artery diameter are not related to body habitus or left ventricular mass. Clin Cardiol. 2014;37:605-9

11. García M, Mulvagh SL, Bairey Merz CN et al. Cardiovascular disease in women: clinical perspectives. Circulation research. 2016;118(8): 1273-93.

12. Macinko J, Guanais FC, Mullachery P et al. Gaps in primary care and health system performance in six Latin American and Caribbean countries. Health Affairs. 2016;35(8):1513-21.
13. Jespersen L, Hvelplund A, Abildstrøm SZ et al. Stable angina pectoris with no obstructive coronary artery disease is associated with increased risks of major adverse cardiovascular events. European heart journal. 2012;33(6):734-44.

14. Gebhard CE, Gebhard C, Maafi F et al. Impact of summer season on prehospital time delays in women and men undergoing primary percutaneous coronary intervention. Sci Total Environ 2019;656:322-30.

15. Rioufol G, Finet G, Ginon I et al. Multiple atherosclerotic plaque rupture in acute coronary syndrome: a three-vessel intravascular ultrasound study. Circulation. 2002;106(7):804-8.

16. Gudnadottir GS, Andersen K, Thrainsdottir IS, et al. Gender differences in coronary angiography, subsequent interventions, and outcomes among patients with acute coronary syndromes. American Heart Journal. 2017;191:65-74. Doi: 10.1016/j.ahj.2017.06.014. 